\title{
THE LOGARITHMIC INTERSECTION BODY
}

\author{
LIJUAN LIU
}

\begin{abstract}
Haberl and Ludwig extended the classical intersection body to $L_{p}$ space, and they showed that the classical intersection body is the limit case of the $L_{p}$ intersection body. In this paper, we introduce the logarithmic intersection body and prove that it is the limit case of the normalized $L_{p}$ intersection body. The affine nature of the logarithmic intersection body operator is demonstrated. Furthermore, a positive answer to the log-Busemann-Petty problem is given.
\end{abstract}

Mathematics subject classification (2010): 52A40, 53A15. volumes.

Keywords and phrases: Star body, radial function, the logarithmic intersection bodies, dual log mixed

\section{REFERENCES}

[1] G. BERCK, Convexity of $L_{p}$-intersection bodies, Adv. Math. 222 (2009), no. 3, 920-936.

[2] K. BÖRÖCZKY, E. LuTwAK, D. YANG, AND G. ZHANG, The log-Brunn-Minkowski inequality, Adv. Math. 231 (2012), no. 3-4, 1974-1997.

[3] K. BÖRÖCZKY, E. LutwaK, D. YANG, AND G. Zhang, The logarithmic Minkowski problem, J. Amer. Math. Soc. 26 (2013), no. 3, 831-852.

[4] K. BöRöczKy, P. Hegedus, G. Zhu, On the discrete logarithmic Minkowski problem, Int. Math. Res. Not. (2016), no. 6, 1807-1838.

[5] S. CAMPI, Convex intersection bodies in three and four dimensions, Mathematika 46 (1999), no. 1, $15-27$.

[6] R. J. GARdner, Intersection bodies and the Busemann-Petty problem, Trans. Amer. Math. Soc. 342 (1994), no. 1, 435-445.

[7] R. J. GARDNER, A positive answer to the Busemann-Petty problem in three dimensions, Ann. of Math. 140 (1994), no.2, 435-447.

[8] R. J. Gardner, Geometric Tomography, 2nd edition, Cambridge Univ. Press, Cambridge, 2006.

[9] R. J. Gardner, D. Hug, W. Weil, And D. Ye, The dual Orlicz-Brunn-Minkowski theory, J. Math. Anal. Appl. 430 (2015), no. 2, 810-829.

[10] R. J. Gardner, A. Koldobsky, T. Schlumprecht, An analytic solution to the Busemann-Petty problem on sections of convex bodies, Ann. of Math. 149 (1999), no. 2, 691-703.

[11] P. Goodey, W. WeIL, Intersection bodies and ellipsoids, Mathematika 42 (1995), no. 2, 295-304.

[12] C. HABERL, $L_{p}$ intersection bodies, Adv. Math. 217 (2008), no. 6, 2599-2624.

[13] C. Haberl, M. Ludwig, A characterization of $L_{p}$ intersection bodies, International Mathematics Research Notices, Art. ID 10548 (2006).

[14] N. J. Kalton, A. Koldobsky, Intersection bodies and L spaces, Adv. Math. 196 (2005), 257275.

[15] A. Koldobsky, Fourier Analysis in Convex Geometry, American Mathematical Society Press, Providence, 2005.

[16] A. Koldobsky, G. Paouris and M. Zymonopoulou, Complex Intersection Bodies, J. Lond. Math. Soc. 88 (2013), 538-562.

[17] M. Ludwig, Intersection bodies and valuations, Amer. J. Math. 128 (2006), no. 6, 1409-1428.

[18] E. LutwaK, Intersection bodies and dual mixed volumes, Adv. Math. 71 (1988), no. 2, 232-261.

[19] C. Saroglou, Remarks on the conjectured log-Brunn-Minkowski inequality, Geom. Dedicata 177 (2015), no. 1, 353-365. 
[20] R. SchneIder, Convex Bodies: The Brunn-Minkowski Theory, Second ed., Cambridge University Press, Cambridge, 2014.

[21] A. Stancu, The discrete planar $L_{0}$-Minkowski problem, Adv. Math., 167 (2002), no. 1, 160-174.

[22] A. STANCU, On the number of solutions to the discrete two dimensional $L_{0}$-Minkowski problem, Adv. Math. 180 (2003), no. 1, 290-323.

[23] A. StancU, The logarithmic Minkowski inequality for non-symmetric convex bodies, Adv. Appl. Math. 73 (2016), 43-58.

[24] W. WANG, L. LiU, The dual log-Brunn-Minkowski inequality, Taiwanese J. Math. 20, (2016), no. 4, 909-919.

[25] W. WANG, N. Zhang, The normalized $L_{p}$ intersection bodies, Math. Inequal. Appl. 21, (2018), no. 2, 353-367.

[26] J. YuAn, W. S. Cheung, $L_{p}$-intersection bodies, J. Math. Anal. Appl. 339 (2008), no. 2, 1431-1439.

[27] C. Zhao, G. Leng, Brunn-Minkowski inequality for mixed intersection bodies, J. Math. Anal. Appl. 301 (2005), no. 1, 115-123.

[28] G. ZHANG, A positive solution to the Busemann-Petty problem in $\mathbb{R}^{4}$, Ann. of Math. 149 (1999), no. 2, 535-543.

[29] B. Zhu, J. Zhou, W. Xu, Dual Orlicz-Brunn-Minkowski theory, Adv. Math. 264 (2014), 700-725.

[30] G. ZHU, The logarithmic Minkowski problem for polytopes, Adv. Math. 262 (2014), 909-931. 